\title{
Twist-3 gluon fragmentation contribution to transversely polarized hyperon production in semi-inclusive deep-inelastic scattering
}

\author{
Riku Ikarashi, ${ }^{a, *}$ Yuji Koike, ${ }^{b}$ Kenta Yabe $^{a}$ and Shinsuke Yoshida ${ }^{c, d}$ \\ ${ }^{a}$ Graduate School of Science and Technology, Niigata University, Ikarashi, Niigata 950-2181, Japan \\ ${ }^{b}$ Department of Physics, Niigata University, Ikarashi, Niigata 950-2181, Japan \\ ${ }^{c}$ Guangdong Provincial Key Laboratory of Nuclear Science, Institute of Quantum Matter, \\ South China Normal University, Guangzhou 510006, China \\ ${ }^{d}$ Guangdong-Hong Kong Joint Laboratory of Quantum Matter, \\ Southern Nuclear Science Computing Center, South China Normal University, Guangzhou 510006, China \\ E-mail: rick.ikatarashi@gmail.com, koike@phys.sc.niigata-u.ac.jp, \\ K.yabe.hadron@gmail.com, shinyoshida85@gmail.com
}

\begin{abstract}
We study the transverse polarization of hyperons produced in semi-inclusive deep inelastic scattering, ep $\rightarrow e \Lambda^{\uparrow} X$, in the collinear twist-3 factorization. This process receives three types of twist- 3 contributions: (i) twist- 3 distribution in the initial proton combined with the transversity fragmentation function (FF) for the hyperon, (ii) Twist-3 quark FFs for the hyperon, and (iii) Twist-3 gluon FFs for the hyperon. In this talk, we present the twist-3 cross section for (iii) in the leading order (LO) with respect to the QCD coupling constant, which completes the LO cross section for this process. Since gluons are ample in the nucleons, this contribution representing multi-gluon correlations in the fragmentation process is potentially as important as other two contributions. This study is relevant for the future Electron-Ion Collider experiment.
\end{abstract}

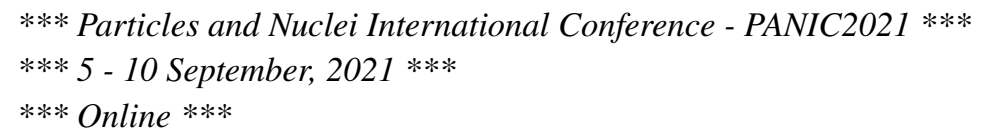

${ }^{*}$ Speaker 


\section{Introduction}

The transverse polarization of hyperons (collectively denoted as $\Lambda$ hereafter) produced in high energy unpolarized proton-proton collision shows a tendency that they become larger in the large rapidity region, typically as large as a few ten \% (e.g. [1]). Since the large transverse polarization was not expected by perturbative QCD at twist-2 level, many efforts have been made to understand its origin for the last several decades.

For a high-energy collision in which particles with large transverse momentum are produced, the cross section can be computed in the framework of the collinear factorization of perturbative QCD. In this framework, the transverse polarization of hyperons appear as a twist-3 observable to which nonperturbative multi-parton correlation functions contribute. The complete leading-order (LO) cross sections contributing to $p p \rightarrow \Lambda^{\uparrow} X$ were derived in [2-6].

In this article, we study the transversely polarized hyperon production in unpolarized electronproton collision $\left(e p \rightarrow e \Lambda^{\uparrow} X\right)$ in the collinear twist-3 factorization, which is relevant for the future Electron-Ion Collider (EIC) experiment in the U.S. Three kinds of twist-3 cross sections contribute to this process: (i) twist-3 unpolarized parton distribution functions (PDFs) in the initial proton convoluted with the twist-2 transversity fragmentation function (FF) for the hyperon, and (ii) twist-3 quark FFs and (iii) twist-3 gluon FFs for the hyperon convoluted with the twist-2 unpolarized PDF in the initial proton. Since the complete LO cross sections for (i) and (ii) were derived in [7], we focus on the contribution (iii) and derive the corresponding cross section, which completes the LO twist-3 cross section for this process [8].

This paper is organized as follows: In Sec. 2, we summarize the twist-3 gluon FFs contributing to $e p \rightarrow e \Lambda^{\uparrow} X$. In Sec. 3, we discuss the twist-3 gluon fragmentation contribution to the spin dependent cross section. Sec. 4 is devoted to a brief summary.

\section{Twist-3 gluon fragmentation functions}

Here we summarize the twist-3 gluon FFs which contribute to $e(\ell)+p(p) \rightarrow e\left(\ell^{\prime}\right)+\Lambda^{\uparrow}\left(P_{h}\right)+X$. They are classified into intrinsic, kinematical and dynamical ones [9]. The intrinsic ones are defined from the 2-gluon correlators as

$$
\begin{aligned}
& \hat{\Gamma}^{\alpha \beta}(z) \\
& =\frac{1}{N^{2}-1} \sum_{X} \int \frac{\mathrm{d} \lambda}{2 \pi} \mathrm{e}^{-\mathrm{i} \lambda / z}\left\langle 0\left|\left([\infty w, 0] F^{w \beta}(0)\right)_{a}\right| h X\right\rangle\left\langle h X\left|\left(F^{w \alpha}(\lambda w)[\lambda w, \infty w]\right)_{a}\right| 0\right\rangle \\
& =M_{h} \epsilon^{P_{h} w S_{\perp}\left\{\alpha_{w} \beta\right\}} \Delta \hat{G}_{3 \bar{T}}(z)+\mathrm{i} M_{h} \epsilon^{\alpha \beta w S_{\perp}} \Delta \hat{G}_{3 T}(z)+\cdots,
\end{aligned}
$$

where $F^{\alpha \beta}$ is a gluon's field strength, $a$ is the color index and $N$ is the color number. $M_{h}$ is the hyperon's mass and $w^{\mu}$ is a lightlike vector satisfying $P_{h} \cdot w=1$. In the twist- 3 accuracy, $P_{h}$ may be treated as a lightlike vector. $[\lambda w, \mu w]=\mathcal{P} \exp \left[\mathrm{i} g \int_{\mu}^{\lambda} \mathrm{d} \tau w \cdot A(\tau w)\right]$ is the gauge link operator which guarantees gauge invariance of the correlation function. Here and below we use the shorthand notation such as $\epsilon^{P_{h} w \alpha \beta} \equiv \epsilon^{\mu \nu \alpha \beta} P_{h \mu} w_{\nu}$. We have written only the twist-3 FFs, $\Delta \hat{G}_{3 \bar{T}}(z)$ and $\Delta \hat{G}_{3 T}(z)$, and omitted other FFs in the equation above. They are real functions and have a support on $0<z<1 . \Delta \hat{G}_{3 \bar{T}}(z)$ is naïvely T-odd, whereas $\Delta \hat{G}_{3 T}(z)$ is naïvely T-even. 
The kinematical FFs are obtained from the derivative of the 2-gluon correlator:

$$
\begin{aligned}
\hat{\Gamma}_{\partial}^{\alpha \beta \gamma}(z) & =\frac{1}{N^{2}-1} \sum_{X} \int \frac{\mathrm{d} \lambda}{2 \pi} \mathrm{e}^{-\mathrm{i} \lambda / z}\left\langle 0\left|\left([\infty w, 0] F^{w \beta}(0)\right)_{a}\right| h X\right\rangle\left\langle h X\left|\left(F^{w \alpha}(\lambda w)[\lambda w, \infty w]\right)_{a}\right| 0\right\rangle \stackrel{\leftarrow}{\partial^{\gamma}} \\
& =-\mathrm{i} \frac{M_{h}}{2} g_{\perp}^{\alpha \beta} \epsilon^{P_{h} w S_{\perp} \gamma} \hat{G}_{T}^{(1)}(z)+\frac{M_{h}}{2} \epsilon^{P_{h} w \alpha \beta} S_{\perp}^{\gamma} \Delta \hat{G}_{T}^{(1)}(z) \\
& -\mathrm{i} \frac{M_{h}}{8}\left(\epsilon^{P_{h} w S_{\perp}\{\alpha} g_{\perp}^{\beta\} \gamma}+\epsilon^{P_{h} w \gamma\{\alpha} S_{\perp}^{\beta\}}\right) \Delta \hat{H}_{T}^{(1)}(z),
\end{aligned}
$$

where $g_{\perp}^{\alpha \beta} \equiv g^{\alpha \beta}-P_{h}^{\alpha} w^{\beta}-P_{h}^{\beta} w^{\alpha}$. The kinematical FFs, $\hat{G}_{T}^{(1)}(z), \Delta \hat{G}_{T}^{(1)}(z)$ and $\Delta \hat{H}_{T}^{(1)}(z)$ are real functions and have a support on $0<z<1$. $\hat{G}_{T}^{(1)}(z)$ and $\Delta \hat{H}_{T}^{(1)}(z)$ are naïvely T-odd and $\Delta \hat{G}_{T}^{(1)}(z)$ is naïvely T-even. They can be also written as the $k_{T}^{2} / M_{h}^{2}$-moment of the TMD FFs. The derivative operator $\overleftarrow{\leftarrow}^{\gamma}$ is used in the following sense:

$$
F^{w \alpha}(\lambda w)[\lambda w, \infty w]|0\rangle \stackrel{\leftarrow}{\partial^{\gamma}} \equiv \lim _{\xi \rightarrow 0} \frac{\mathrm{d}}{\mathrm{d} \xi_{\gamma}} F^{w \alpha}(\lambda w+\xi)[\lambda w+\xi, \infty w+\xi]|0\rangle
$$

The dynamical FFs are defined from the 3-gluon correlators. The contraction with two structure constants for the color $\mathrm{SU}(N), f_{a b c}$ and $d_{a b c}$, defines

$$
\begin{aligned}
& \hat{\Gamma}_{F A}^{\alpha \beta \gamma}\left(\frac{1}{z_{1}}, \frac{1}{z_{2}}\right) \\
& =\frac{-\mathrm{i} f_{a b c}}{N^{2}-1} \sum_{X} \iint \frac{\mathrm{d} \lambda}{2 \pi} \frac{\mathrm{d} \mu}{2 \pi} \mathrm{e}^{-\mathrm{i} \lambda / z_{1}} \mathrm{e}^{-\mathrm{i} \mu\left(1 / z_{2}-1 / z_{1}\right)}\left\langle 0\left|F_{b}^{w \beta}(0)\right| h X\right\rangle\left\langle h X\left|F_{a}^{w \alpha}(\lambda w) g F_{c}^{w \gamma}(\mu w)\right| 0\right\rangle \\
& =-M_{h}\left(\hat{N}_{1}\left(\frac{1}{z_{1}}, \frac{1}{z_{2}}\right) g_{\perp}^{\alpha \gamma} \epsilon^{P_{h} w S_{\perp} \beta}+\hat{N}_{2}\left(\frac{1}{z_{1}}, \frac{1}{z_{2}}\right) g_{\perp}^{\beta \gamma} \epsilon^{P_{h} w S_{\perp} \alpha}-\hat{N}_{2}\left(\frac{1}{z_{2}}-\frac{1}{z_{1}}, \frac{1}{z_{2}}\right) g_{\perp}^{\alpha \beta} \epsilon^{P_{h} w S_{\perp} \gamma}\right), \\
& \hat{\Gamma}_{F S}^{\alpha \beta \gamma}\left(\frac{1}{z_{1}}, \frac{1}{z_{2}}\right) \\
& =\frac{d_{a b c}}{N^{2}-1} \sum_{X} \iint \frac{\mathrm{d} \lambda}{2 \pi} \frac{\mathrm{d} \mu}{2 \pi} \mathrm{e}^{-\mathrm{i} \lambda / z_{1}} \mathrm{e}^{-\mathrm{i} \mu\left(1 / z_{2}-1 / z_{1}\right)}\left\langle 0\left|F_{b}^{w \beta}(0)\right| h X\right\rangle\left\langle h X\left|F_{a}^{w \alpha}(\lambda w) g F_{c}^{w \gamma}(\mu w)\right| 0\right\rangle \\
& =-M_{h}\left(\hat{O}_{1}\left(\frac{1}{z_{1}}, \frac{1}{z_{2}}\right) g_{\perp}^{\alpha \gamma} \epsilon^{P_{h} w S_{\perp} \beta}+\hat{O}_{2}\left(\frac{1}{z_{1}}, \frac{1}{z_{2}}\right) g_{\perp}^{\beta \gamma} \epsilon^{P_{h} w S_{\perp} \alpha}+\hat{O}_{2}\left(\frac{1}{z_{2}}-\frac{1}{z_{1}}, \frac{1}{z_{2}}\right) g_{\perp}^{\alpha \beta} \epsilon^{P_{h} w S_{\perp} \gamma}\right),
\end{aligned}
$$

where we have suppressed the gauge link operator. The four dynamical FFs, $\hat{N}_{1,2}\left(\frac{1}{z_{1}}, \frac{1}{z_{2}}\right)$ and $\hat{O}_{1,2}\left(\frac{1}{z_{1}}, \frac{1}{z_{2}}\right)$, are complex functions and have a support on $\frac{1}{z_{2}}>1$ and $\frac{1}{z_{2}}>\frac{1}{z_{1}}>0$. Their real parts are naïvely T-even and the imaginary parts are naïvely T-odd, the latter being the source of the hyperon polarization.

One also needs to introduce another type of dynamical FFs which are defined from the quarkantiquark-gluon correlators:

$$
\begin{aligned}
\tilde{\Delta}_{i j}^{\alpha}\left(\frac{1}{z_{1}}, \frac{1}{z_{2}}\right) & =\frac{1}{N} \sum_{X} \iint \frac{\mathrm{d} \lambda}{2 \pi} \frac{\mathrm{d} \mu}{2 \pi} \mathrm{e}^{-\mathrm{i} \lambda / z_{1}} \mathrm{e}^{-\mathrm{i} \mu\left(1 / z_{2}-1 / z_{1}\right)}\left\langle 0\left|g F_{a}^{w \alpha}(\mu w)\right| h X\right\rangle\left\langle h X\left|\bar{\psi}_{j}(\lambda w) t^{a} \psi_{i}(0)\right| 0\right\rangle \\
& =M_{h}\left(\epsilon^{\alpha P_{h} w S_{\perp}}\left(\boldsymbol{P}_{h}\right)_{i j} \tilde{D}_{F T}\left(\frac{1}{z_{1}}, \frac{1}{z_{2}}\right)+\mathrm{i} S_{\perp}^{\alpha}\left(\gamma_{5} \boldsymbol{P}_{h}\right)_{i j} \tilde{G}_{F T}\left(\frac{1}{z_{1}}, \frac{1}{z_{2}}\right)\right)
\end{aligned}
$$


where $t^{a}$ is the generators of the $\mathrm{SU}(N) . \tilde{D}_{F T}\left(\frac{1}{z_{1}}, \frac{1}{z_{2}}\right)$ and $\tilde{G}_{F T}\left(\frac{1}{z_{1}}, \frac{1}{z_{2}}\right)$ are, in general, complex functions and have a support on $\frac{1}{z_{1}}>0, \frac{1}{z_{2}}>0$ and $\frac{1}{z_{1}}-\frac{1}{z_{2}}>1$. Their real parts are naïvely T-even, while the imaginary parts are naïvely T-odd.

\section{Twist-3 gluon FFs contribution to the cross section}

The twist-3 gluon FF contribution to $e p \rightarrow e \Lambda^{\uparrow} X$ is diagrammatically shown in Fig.1. We
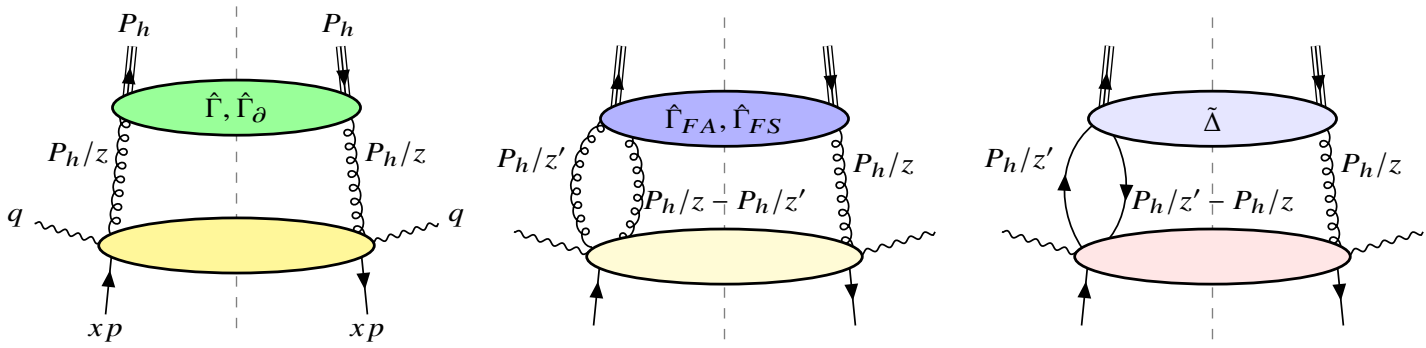

Figure 1: Generic diagrams giving rise to the twist-3 gluon fragmentation contribution to $e p \rightarrow e \Lambda^{\uparrow} X$. The upper blobs represent the twist-3 gluon FFs and the lower blobs represent the partonic hard parts. We have omitted the distribution part and the electron lines in the each diagram.

work in the hadron frame in which the momenta of the initial proton and the virtual photon are collinear [10]. Following the formalism developed for $p p \rightarrow \Lambda^{\uparrow} X$ in [6], we could obtain the LO cross section as

$$
\begin{aligned}
& \frac{\mathrm{d}^{6} \sigma}{\mathrm{d} x_{b j} \mathrm{~d} Q^{2} \mathrm{~d} z_{f} \mathrm{~d} q_{\mathrm{T}}^{2} \mathrm{~d} \phi \mathrm{d} \chi} \\
& =\frac{\alpha_{e m}^{2} \alpha_{s} M_{h}}{16 \pi^{2} x_{b j}^{2} S_{e p}^{2} Q^{2}} \sum_{k=1,2,3,4,8,9} \mathcal{A}_{k}(\phi-\chi) \mathcal{S}_{k}\left(\Psi_{s}\right) \iint \mathrm{d} x \mathrm{~d}\left(\frac{1}{z}\right) \frac{z^{3}}{x} f_{1}(x) \delta\left(\frac{q_{T}^{2}}{Q^{2}}-\left(1-\frac{1}{\hat{x}}\right)\left(1-\frac{1}{\hat{z}}\right)\right) \\
& \left\{\hat{G}_{T}^{(1)}(z) \hat{\sigma}_{G}^{k}+\Delta \hat{H}_{T}^{(1)}(z) \hat{\sigma}_{H}^{k}\right. \\
& +\int \mathrm{d}\left(\frac{1}{z^{\prime}}\right)\left[\frac{1}{1 / z-1 / z^{\prime}} \mathfrak{J}\left(\hat{N}_{1}\left(\frac{1}{z^{\prime}}, \frac{1}{z}\right) \hat{\sigma}_{N 1}^{k}+\hat{N}_{2}\left(\frac{1}{z^{\prime}}, \frac{1}{z}\right) \hat{\sigma}_{N 2}^{k}+\hat{N}_{2}\left(\frac{1}{z}-\frac{1}{z^{\prime}}, \frac{1}{z}\right) \hat{\sigma}_{N 33}^{k}\right)\right. \\
& \left.+\frac{1}{z}\left(\frac{1}{1 / z-1 / z^{\prime}}\right)^{2} \mathfrak{J}\left(\hat{N}_{1}\left(\frac{1}{z^{\prime}}, \frac{1}{z}\right) \hat{\sigma}_{D N 1}^{k}+\hat{N}_{2}\left(\frac{1}{z^{\prime}}, \frac{1}{z}\right) \hat{\sigma}_{D N 2}^{k}+\hat{N}_{2}\left(\frac{1}{z}-\frac{1}{z^{\prime}}, \frac{1}{z}\right) \hat{\sigma}_{D N 3}^{k}\right)+\{N \rightarrow O\}\right] \\
& +\int \mathrm{d}\left(\frac{1}{z^{\prime}}\right) \frac{2}{C_{F}}\left[\mathfrak { J } \tilde { D } _ { F T } ( \frac { 1 } { z ^ { \prime } } , \frac { 1 } { z ^ { \prime } } - \frac { 1 } { z } ) \left(\hat{\sigma}_{D F 1}^{k}+\frac{1}{z} \frac{1}{1 / z-1 / z^{\prime}} \hat{\sigma}_{D F 2}^{k}+\frac{z^{\prime}}{z} \hat{\sigma}_{D F 3}^{k}\right.\right. \\
& \left.\left.\left.+\frac{1}{1-\left(1-q_{T}^{2} / Q^{2}\right) z_{f} / z^{\prime}} \hat{\sigma}_{D F 4}^{k}+\frac{1}{1-\left(1-q_{T}^{2} / Q^{2}\right) z_{f}\left(1 / z-1 / z^{\prime}\right)} \hat{\sigma}_{D F 5}^{k}\right)+\{D \rightarrow G\}\right]\right\},
\end{aligned}
$$

where $\{N \rightarrow O\}$ represents terms obtained by replacing every $N$ in the same $[\cdots]$ by $O$, and likewise for $\{D \rightarrow G\}$. $f_{1}(x)$ is the unpolarized distribution function and $\hat{\sigma}^{k}$ s represent the partonic hard scattering parts. $\hat{x}$ and $\hat{z}$ are givein by $\hat{x} \equiv x_{b j} / x, \hat{z} \equiv z_{f} / z$ and the Lorentz invariants, $S_{e p}$, $Q^{2}, x_{b j}, z_{f}$ and $q_{T}$, are defined by $S_{e p}=(p+\ell)^{2}, Q^{2}=-q^{2}=-\left(\ell-\ell^{\prime}\right)^{2}, x_{b j}=\frac{Q^{2}}{2 p \cdot q}$, $z_{f}=\frac{p \cdot P_{h}}{p \cdot q}$ and $q_{T}=\sqrt{-q_{t}^{2}}\left(q_{t}^{\mu}=q^{\mu}-\frac{P_{h} \cdot q}{p \cdot P_{h}} p^{\mu}-\frac{p \cdot q}{p \cdot P_{h}} P_{h}^{\mu}\right)$. The $\chi$ and $\phi$ are, respectively, the 
azimuthal angles of the hadron plane and the lepton plane in the hadron frame. $\mathcal{A}_{k}(\varphi)(\varphi=\phi-\chi$, $k=1, \cdots, 4,8,9)$ are defined as [10] $\mathcal{A}_{1}(\varphi)=1+\cosh ^{2} \psi, \mathcal{A}_{2}(\varphi)=-2, \mathcal{A}_{3}(\varphi)=-\cos \varphi \sinh 2 \psi$, $\mathcal{A}_{4}(\varphi)=\cos 2 \varphi \sinh ^{2} \psi, \mathcal{A}_{8}(\varphi)=-\sin \varphi \sinh 2 \psi, \mathcal{A}_{9}(\varphi)=\sin 2 \varphi \sinh ^{2} \psi$, which carry the azimuthal dependence of the cross section. $\mathcal{S}_{1,2,3,4}=\sin \Psi_{s}$ and $\mathcal{S}_{8,9}=\cos \Psi_{s}$, where $\Psi_{s}$ denotes the azimuthal angle of the transverse spin vector of the final hyperon. According to the formalism developed in [6], intrinsic, kinematical, and the dynamical twist-3 FFs as well as the derivative of kinematical FFs appear in the cross section. By using the QCD equation-of-motion relations and the Lorentz invariance relations [9], one can eliminate the intrinsic FF, $\Delta \hat{G}_{3 \bar{T}}(z)$, and the derivative of the kinematical FFs, $\partial \hat{G}_{T}^{(1)}(z) / \partial(1 / z)$ and $\partial \Delta \hat{H}_{T}^{(1)}(z) / \partial(1 / z)$, in favor of the other twist-3 FFs. Therefore the final form of the cross section can be written in terms of the twist-3 gluon FFs, $\hat{G}_{T}^{(1)}$, $\Delta \hat{H}_{T}^{(1)}, \mathfrak{J} \hat{N}_{1,2}, \mathfrak{J} \hat{O}_{1,2}, \mathfrak{J} \tilde{D}_{F T}$ and $\mathfrak{J} \tilde{G}_{F T}$ as in (7). The detail of the calculation will be reported in [8].

\section{Summary}

In this work, we have derived the twist-3 gluon fragmentation contribution to the transversely polarized hyperon production in semi-inclusive deep inelastic scattering, $e p \rightarrow e \Lambda^{\uparrow} X$, within the collinear twist-3 factorization. Together with the result in [7], this has completed the LO cross section for this process.

\section{Acknowledgments}

This work has been supported by the establishment of Niigata university fellowships towards the creation of science technology innovation (R.I.), the Grant-in-Aid for Scientific Research from the Japanese Society of Promotion of Science under Contract Nos. 19K03843 (Y.K.) and 18J11148 (K.Y.), National Natural Science Foundation in China under grant No. 11950410495, Guangdong Natural Science Foundation under No. 2020A1515010794 and research startup funding at South China Normal University.

\section{References}

[1] G. Bunce et al. , Phys. Rev. Lett. 36, 1113 (1976).

[2] Y. Kanazawa and Y. Koike, Phys. Rev. D 64, 034019 (2001).

[3] J. Zhou, F. Yuan, and Z. T. Liang, Phys. Rev. D 78, 114008 (2008).

[4] Y. Koike, K. Yabe, and S. Yoshida, Phys. Rev. D 92, 094011 (2015).

[5] Y. Koike, A. Metz, D. Pitonyak, K. Yabe, and S. Yoshida, Phys. Rev. D 95, 114013 (2017).

[6] Y. Koike, K. Yabe and S. Yoshida, Phys. Rev. D 104, 054023 (2021).

[7] Y. Koike, K. Takada, S. Usui, K. Yabe and S. Yoshida, in preparation.

[8] R. Ikarashi, Y. Koike, K. Yabe and S. Yoshida, in preparation.

[9] Y. Koike, K. Yabe and S. Yoshida, Phys. Rev. D 101, 054017 (2020).

[10] R. Meng, F. I. Olness and D. E. Soper, Nucl. Phys. B 371, 79 (1992). 